\title{
Transfer of meropenem in the ex vivo human placenta perfusion model
}

\author{
MICHAEL HNAT \& ROGER E. BAWDON \\ Division of Maternal-Fetal Medicine, Department of Obstetrics and Gynecology, The University of Texas, Southwestern \\ Medical Center at Dallas, USA
}

\begin{abstract}
Objectives. To determine maternal-fetal transplacental passage of meropenem in the ex vivo human perfusion model. Study design. Term placentae $(n=6)$ were collected immediately after delivery. A single cotyledon was localized, perfused and stabilized with physiologic Eagles minimal essential medium containing 3\% bovine albumin and heparin as described by Chalier (Chalier JC. Criteria for evaluating perfusion experiments and presentation results. Contrib Gynecol Obstet 1985; 13:32-39). Meropenem was added to the maternal medium in concentrations similar to maternal serum peak and trough levels, then perfused through the maternal circulation of the cotyledon. To assess transfer and accumulation, fluid aliquots from both the maternal and fetal compartments were collected over an hour at defined intervals in an open and closed system. Antipyrine ${ }^{14} \mathrm{C}$ was added to the medium in order to calculate the transport fraction and clearance indexes. Meropenem and antipyrine ${ }^{14} \mathrm{C}$ concentrations were determined by High-pressure Liquid Chromatography and liquid scintillation, respectively.

Results. Mean antipyrine transport fraction was $2.33+0.25$. Maternal and fetal mean meropenem peak concentrations were $54.3+3.3 \mu \mathrm{g} / \mathrm{ml}$ and $2.2+0.18 \mu \mathrm{g} / \mathrm{ml}$, respectively. Whereas, maternal and fetal mean trough concentrations were $12.7+1.3 \mu \mathrm{g} / \mathrm{ml}$ and $0.41+0.10 \mu \mathrm{g} / \mathrm{ml}$, respectively. Mean peak clearance index was $0.077+0.007$ and the mean trough was $0.052+0.015$. Mean accumulation for the peak and trough concentrations of meropenem were 0.9 and $2.95 \mu \mathrm{g} / \mathrm{ml}$, respectively.

Conclusions. Transplacental passage of meropenem was incomplete in the ex vivo human placental perfusion model. Accumulation was also noted in the fetal compartment
\end{abstract}

Keywords: Meropenem, antibiotic, fetus, placental perfusion and pregnancy

\section{Condensation}

Incomplete transplacental passage and accumulation of meropenem were noted in the ex vivo human placental perfusion model.

\section{Introduction}

Meropenem is a synthetic carbapenem $\beta$-lactam antibiotic with a broad antimicrobial activity against gram-negative, gram-positive and anaerobic microorganisms. It is as effective as imipenem/cilastatin and is more effective than cephalosporin-based combination in the treatment of a range of serious infections. Resistance to meropenem is uncommon [1-3].
Use of this particular antibiotic is increasing secondary to microbial resistance to other antibiotics. In addition, meropenem has been administered to pregnant women. Like many other medications, information regarding pharmacokinetics is limited for meropenem in pregnancy [4,5]. Our study objective was to quantify the maternal to fetal transplacental passage of meropenem in the ex vivo human placental perfusion model.

\section{Material and methods}

Term placentae were collected immediately after delivery from either cesarean sections or vaginal deliveries in accordance with the University of Texas

\footnotetext{
Correspondence: Michael Hnat, D.O., UT Southwestern Medical Center, 5323 Harry Hines Blvd., Dallas, TX 75390-9032, USA. Tel: $214 / 6484746$. Fax: 214/648 4763. E-mail: michael.hnat@utsouthwestern.edu

Presented at the Annual Scientific Meeting and Symposium of the Infectious Diseases Society for Obstetrics and Gynecology, San Diego, California, August 5-7, 2004
} 
Southwestern Medical Center Institutional Review Board for Human Studies' guidelines and were transported to the laboratory in normal saline immediately after delivery.

The single cotyledon placental perfusion system was used as described by Chalier [6]. A fetal artery and vein on the chorionic plate was cannulated with $3.0 \mathrm{~F}$ and $5.0 \mathrm{~F}$ catheters, respectively (Figure 1 ). The fetal circulation of the selected cotyledon was gently perfused with Eagles minimal essential media plus $3 \%$ bovine albumin and 1.0 units $/ \mathrm{ml}$ of heparin (Sigma Chemical Co., St. Louis, MO).

The localized cotyledon and surrounding placental tissue were transferred to a temperature-controlled chamber $\left(37^{\circ} \mathrm{C}\right)$ where the fetal circulation was perfused in a closed-system at a rate between 4.5 and $5 \mathrm{~mL} / \mathrm{min}$ for 20 minutes to remove any residual blood and to stabilize the perfusion pressure between 35 and $45 \mathrm{mmHg}$ (Figure 2). Prior to perfusing, two 18-guage needles were inserted into the intervillous space of the cotyledon to reestablish the maternal circulation (Figure 3). Maternal flow rate was $17 \mathrm{~mL} / \mathrm{min}$. Those placental cotyledons, which failed to reach a stable baseline pressure or found to have a vascular leak, were discarded.

The maternal and fetal compartments consisted of $150 \mathrm{~mL}$ of Eagle's minimal essential medium ( $\mathrm{pH} 7.2$ -7.4 ), which were aerated with $95 \%$ oxygen and $5 \%$ carbon dioxide and were continually mixed by a magnetic stirring bar. Transfer studies were performed with meropenem (Zeneca Pharmaceuticals, Wilmington, DE). Meropenem was added to the maternal medium in concentrations similar to serum peak and trough levels. Experiments were first conducted in an open-open system to determine transport fraction, then in a closed-closed recirculation system to determine accumulation. The clearance index of meropenem was also calculated. Fluid aliquots from both the maternal and fetal compartments were collected for analysis.

A total of 6 placentae met criteria to be included in the study. A trough transfer study for meropenem was performed in one placenta and a peak study in another. Both, peak and trough transfer studies were conducted in the remaining four. Studies were completed first in an open system for 1 hour and then in a closed system. For example, in the 4 placentae, trough studies were performed in an open system and then in a closed system, followed by peak studies in an open system and then in a closed one.

Lastly, transport fraction and clearance indexes were determined for each placenta by means of the ${ }^{14} \mathrm{C}$ antipyrine reference method of Challier [6]. Placentae were studied in a nonrecirculating (open) system for 60 minutes to determine transport fraction of ${ }^{14} \mathrm{C}$ antipyrine. Greater than $40 \%$ maternal-fetal transfer of antipyrine is an indication of a successful maternal-fetal match.

Meropenem and antipyrine ${ }^{14} \mathrm{C}$ concentrations were measured by High-pressure Liquid Chromatography (HPLC) and liquid scintillation, respectively. Reproducibility studies on the meropenem assay were performed.

The HPLC analysis of meropenem was carried out on all Waters Associate instruments (Medford, MA). The meropenem standards were prepared from laboratory assay powder provided by Zeneca Pharmaceuticals. The HPLC assay of meropenem consisted of preparation of a standard curve to breach the therapeutic range of the drug. All specimens and standards were extracted in aliquots of $0.5 \mathrm{~mL}$. To each tube was added $2.5 \mathrm{~mL}$ of acetonitrile to precipitate blood, serum and other proteins. All samples were centrifuged at $800 \times \mathrm{g}$ for 10 minutes and the supernatant was decanted into a clean $10 \mathrm{~mm} \times 100 \mathrm{~mm}$ screw-capped test tube. Five milliliters of methylene chloride was then added to each tube. The tubes were mixed on a vortex mixer and were again centrifuged at $800 \times \mathrm{g}$. The upper aqueous layer was removed and placed in HPLC micro tubes and placed in a Waters $717+$ autosampler. An injection of $20 \mathrm{~mL}$ was made. The HPLC mobile phase consisted of $95 \%$ mmole ammonium acetate and $5 \%$ acetonitrile $(\mathrm{pH} 4.0)$.
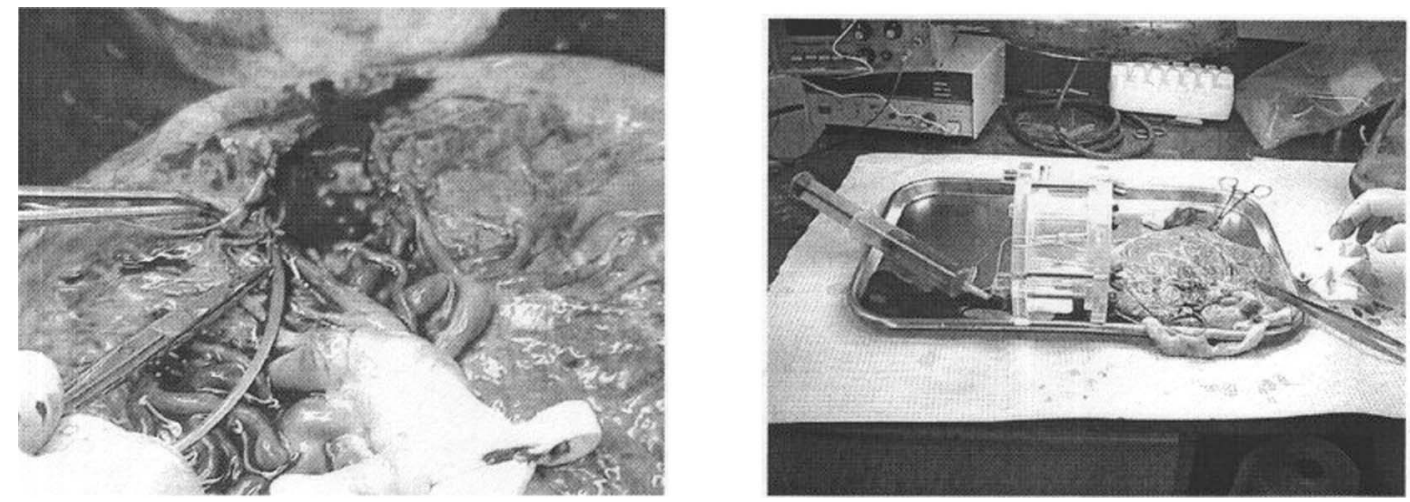

Figure 1. Fetal circulation. (a) Cannulization of vein. (b) Establishing fetal circulation. 


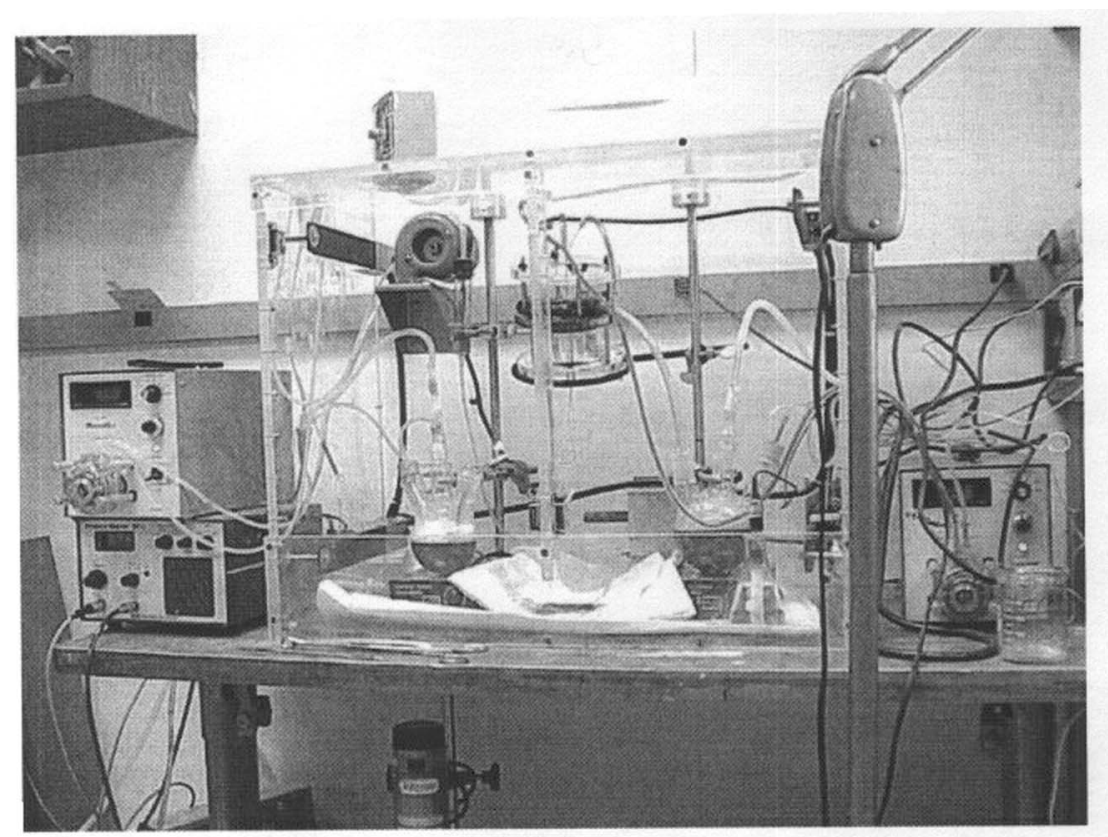

Figure 2. Placental perfusion model.
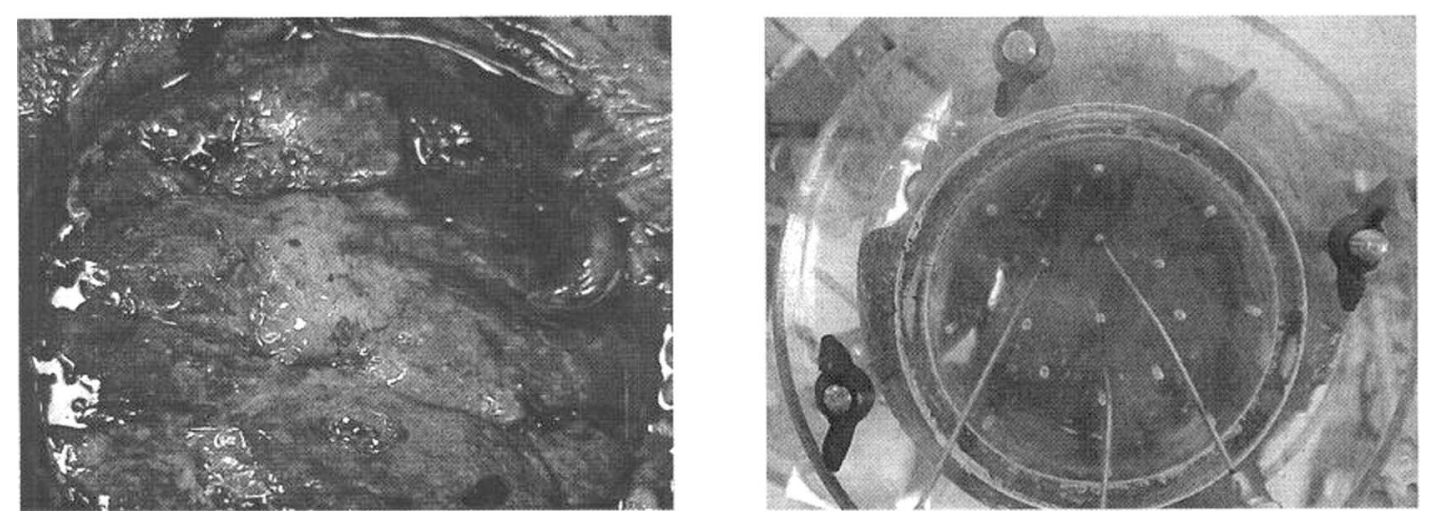

Figure 3. Maternal circulation. (a) Perfused cotyledon. (b) 18 gauge probes for maternal perfusate.

The HPLC column used was Waters $\mu$-Bond a-Pak $\mathrm{C}_{18}$ with a flow rate of $1.5 \mathrm{~mL} / \mathrm{min}$. The HPLC pump was a Waters model 510 . The Waters 481 detector settings were a wavelength of $298 \mu \mathrm{m}$ with a sensitivity setting of 0.01-0.05 absorbance units. The retention time was about 7 minutes and the peak height was recorded on a Kipp and Zonan (Holland) $10 \mathrm{mV}$ recorder. Using least squares regression analysis and the correlation coefficient of the standard curve all specimens were quantified and reported. Reproducibility studies were done on meropenem over a 3 day period to determine the within batch and between batch reproducibility.

\section{Results}

For the HPLC determination of meropenem concentrations, reproducibility studies provided values with a minimum sensitivity of $0.2 \mu \mathrm{g} / \mathrm{mL}$; and, a within batch and between batch reproducibility of $>90 \%$. Maternal and fetal Meropenem concentrations for the maternal and fetal perfusate are listed in Table I. For the maternal perfusate, the mean peak and trough levels and standard error were $54.3 \mu \mathrm{g} /$ $\mathrm{mL} \pm 3.3$ and $12.7 \pm 1.3 \mu \mathrm{g} / \mathrm{mL}$, respectively and for the fetal, they were $2.2 \pm 1.8 \mu \mathrm{g} / \mathrm{mL}$ and $0.44 \pm$ $0.10 \mu \mathrm{g} / \mathrm{mL}$. The clearance index and accumulation for both peak and trough levels are shown in Table II. The mean transport factor for antipyrine ${ }^{14} \mathrm{C}$ was $2.33 \pm 0.25$.

\section{Discussion}

The pharmacokinetics and pharmacodynamics of meropenem have been described in patients who are healthy or critically ill, who have renal or hepatic 
Table I. Meropenem concentrations.

\begin{tabular}{lccccc}
\hline & \multicolumn{2}{c}{ Peak Concentration $(\mu \mathrm{g} / \mathrm{mL})$} & & \multicolumn{2}{c}{ Trough Concentration $(\mu \mathrm{g} / \mathrm{mL})$} \\
\cline { 2 - 3 } \cline { 5 - 6 } Placenta \# & Maternal & Fetal & Maternal & Fetal \\
\hline 1 & 63.7 & 1.97 & 17.1 & 0.68 \\
2 & 53.3 & 2.80 & 13.1 & 0.57 \\
3 & - & 1.80 & 12.3 & 0.5 \\
5 & 59.8 & 2.45 & 9.1 & 0.36 \\
6 & 45.8 & 2.17 & - & - \\
\hline
\end{tabular}

Table II. Meropenem clearance index and accumulation.

\begin{tabular}{|c|c|c|c|c|}
\hline \multirow[b]{2}{*}{ Placenta \# } & \multicolumn{2}{|c|}{ Clearance Index } & \multicolumn{2}{|c|}{ Accumulation $(\mu \mathrm{g} / \mathrm{mL})$} \\
\hline & Peak & Trough & Peak & Trough \\
\hline 1 & 0.062 & 0.078 & 3.3 & 1.0 \\
\hline 2 & 0.062 & 0.067 & 3.5 & 1.4 \\
\hline 3 & - & 0.011 & - & 1.5 \\
\hline 4 & 0.085 & 0.082 & 5.9 & 0.7 \\
\hline 5 & 0.096 & 0.020 & 2.8 & 0.7 \\
\hline 6 & 0.082 & - & 5.4 & - \\
\hline
\end{tabular}

impairment and who are elderly or young $[2,7,8]$. However, little is known regarding its pharmacokinetics and safety in the mother and the fetus during pregnancy $[4,5]$. Meropenem is listed as Category B in pregnancy [5]. In other words, no adequate or well-controlled studies have been carried out in pregnant women. No fetal harm was noted in rats and cynomolgus monkeys at doses of 1.8 and 3.7 times the human dose [5]. One abstract was available that described the efficacy and safety of meropenem during pregnancy $(n=13)$ and during the puerperal period $(n=11)$ [9]. Our study provides initial data regarding transplacental passage of meropenem in the ex vivo human model.

Meropenem is similar in structure to imipenem, another carbapenem beta-lactam antibiotic. Imipenem's pharmacokinetics is known to change during pregnancy [8]. When compared to healthy, nonpregnant women, Imipenem's volume of distribution was larger, clearance was accelerated and peak levels were significantly lower in those who were pregnant [8]. Meropenem mean values for peak plasma concentrations and volume of distribution after $1 \mathrm{~g}$ doses are known for a healthy individual and are 54.8 to $61.6 \mu \mathrm{g} / \mathrm{mL}$ and 12.5 to $23 \mathrm{~L}$, respectively [3]. These values during pregnancy are unknown.

Heikkilä and co-workers demonstrated transplacental passage of imipenem and noted therapeutic concentrations in the fetal compartments late in pregnancy [8]. In our study, transplacental passage of meropenem was noted to occur in the ex vivo model. However, concentrations of meropenem in the fetal effluence were less than maternal levels and were below therapeutic levels. Therefore, placental transfer was incomplete. The low clearance index obtained in this study might be explained by the solubility and molecular weight of meropenem. Most drugs cross the placenta by simple diffusion. Rate of diffusion is dependent upon solubility, molecular weight and concentration of the free drug. Hydrophilic substances cross the placenta slower than lipophilic ones. Meropenem is hydrophilic with a molecular weight of $437.52 \mathrm{~d}$ [3]. Further research is needed in vivo to determine both fetal and maternal concentrations of meropenem.

Normal physiologic changes of pregnancy may alter the pharmacokinetics of meropenem not apparent in our ex vivo model. In pregnancy, blood volume and glomerular filtration rate increase 40$50 \%$. Because meropenem and its inactive metabolite are excreted by the kidney through glomerular filtration and tubular secretion serum concentrations may be decreased in pregnancy $[3,8]$.

Accumulation of meropenem in the fetal compartment was noted in the closed system. Minimal accumulation was noted at a maternal peak and trough concentrations. Fetal accumulation increased with maternal meropenem concentrations but remained below the therapeutic levels.

Our study suggests that meropenem crosses the placenta incompletely with lower fetal concentrations than maternal. In addition, the placental clearance and fetal accumulation of meropenem are low at both peak and trough levels. Further investigation of 
meropenem in vivo is needed to determine both the maternal and fetal pharmacokinetics.

\section{Acknowledgments}

I would like to thank those at Parkland Memorial Hospital who aided in obtaining the placentae for this study, especially the unit clerks, midwives, nurses and residents.

\section{References}

1. Hurst M, Lamb HM. Meropenem. A review of its use in patients in intensive care. Drugs 2000;59(3):652-680.

2. Drusano GL, Hutchison M. The pharmacokinetics of meropenem. Scand J Infect Dis 1995;96 Supp:11-16.
3. Hemsell DL, Martens MG, Faro S, Gall S, McGregor JA. Clin Infect Dis 1997;24:S222-S230.

4. Physicians' Desk Reference. 58th ed. Montvale, NJ: Thomson PDR; 2004. pp 674-678.

5. Kitzes-Cohen R, Farin D, Piva G, De Myttenaere-Bursztein SA. Int J Antimicrob Agents. $2002 \mathrm{Feb} ; 19(2): 105-110$.

6. Chalier JC. Criteria for evaluating perfusion experiments and presentation results. Contrib Gynecol Obstet 1985;13:32-39.

7. Leroy A, Fillastre JP, Borsa-Lebas F, Etienne I, Humbert G. Pharmacokinetics of meropenem (ICI 194,660) and its metabolite (ICI 213,689) in healthy subjects and in patients with renal impairment. Antimicrob Agents Chemother 1992;36(12):2794-2798.

8. Heikkilä A, Renkonen OK, Erkkola R. Pharmacokinetics and transplacental passage of imipenem during pregnancy. Antimicrob Agents Chemother 1992;36(12):2652-2655.

9. Chimura $\mathrm{T}$, Murayama $\mathrm{K}$, Oda $\mathrm{T}$, Igarashi $\mathrm{Y}$, Morisaki $\mathrm{N}$, Hirayama $\mathrm{T}$, Kihara $\mathrm{K}$. Clinical effects of meropenem on infectious diseases in obstetrics and gynecology [in Japanese]. Jpn J Antibiot 2001;54(1):1-7. English abstract. 


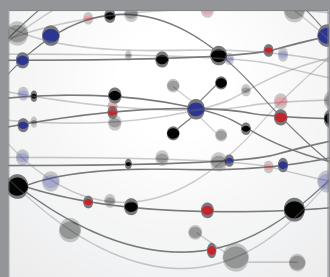

The Scientific World Journal
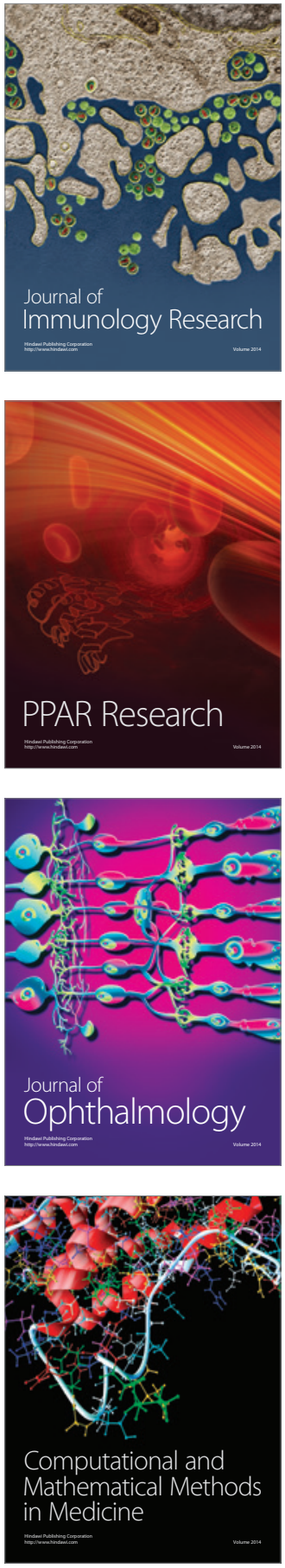

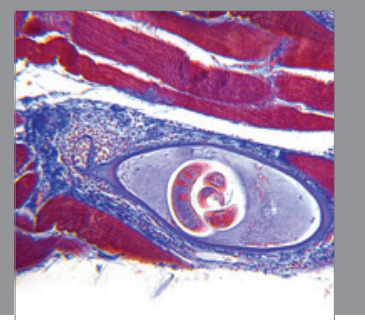

Gastroenterology

Research and Practice
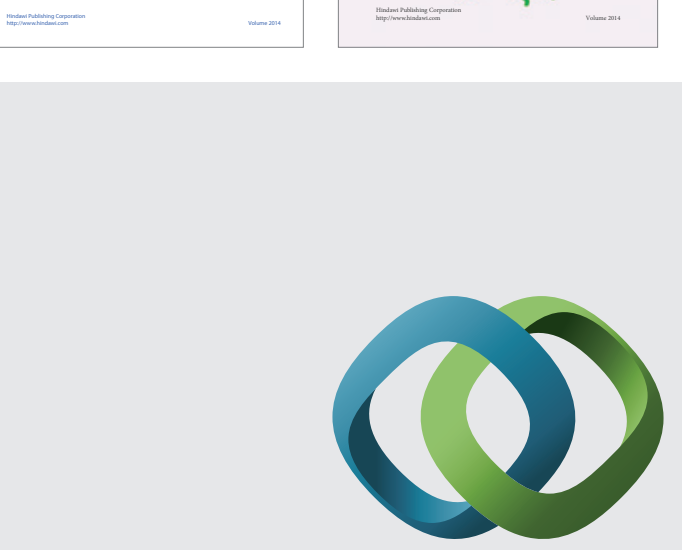

\section{Hindawi}

Submit your manuscripts at

http://www.hindawi.com
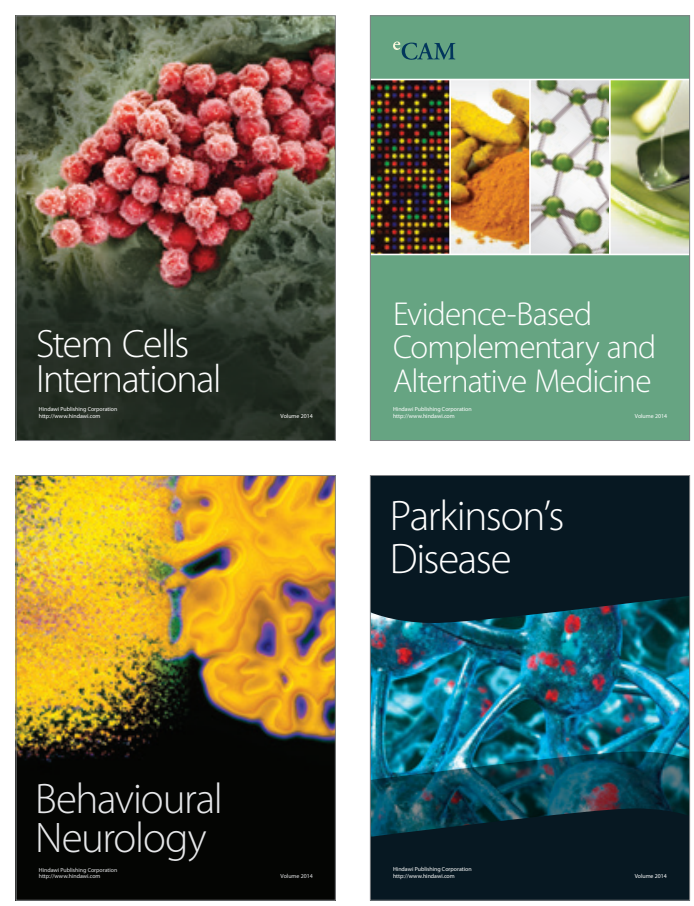

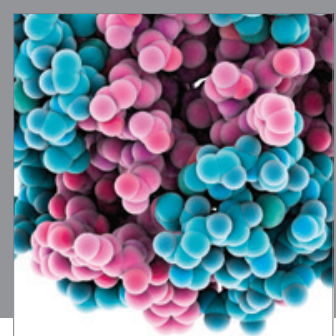

Journal of
Diabetes Research

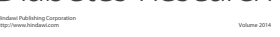

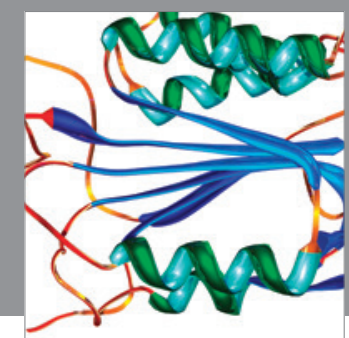

Disease Markers
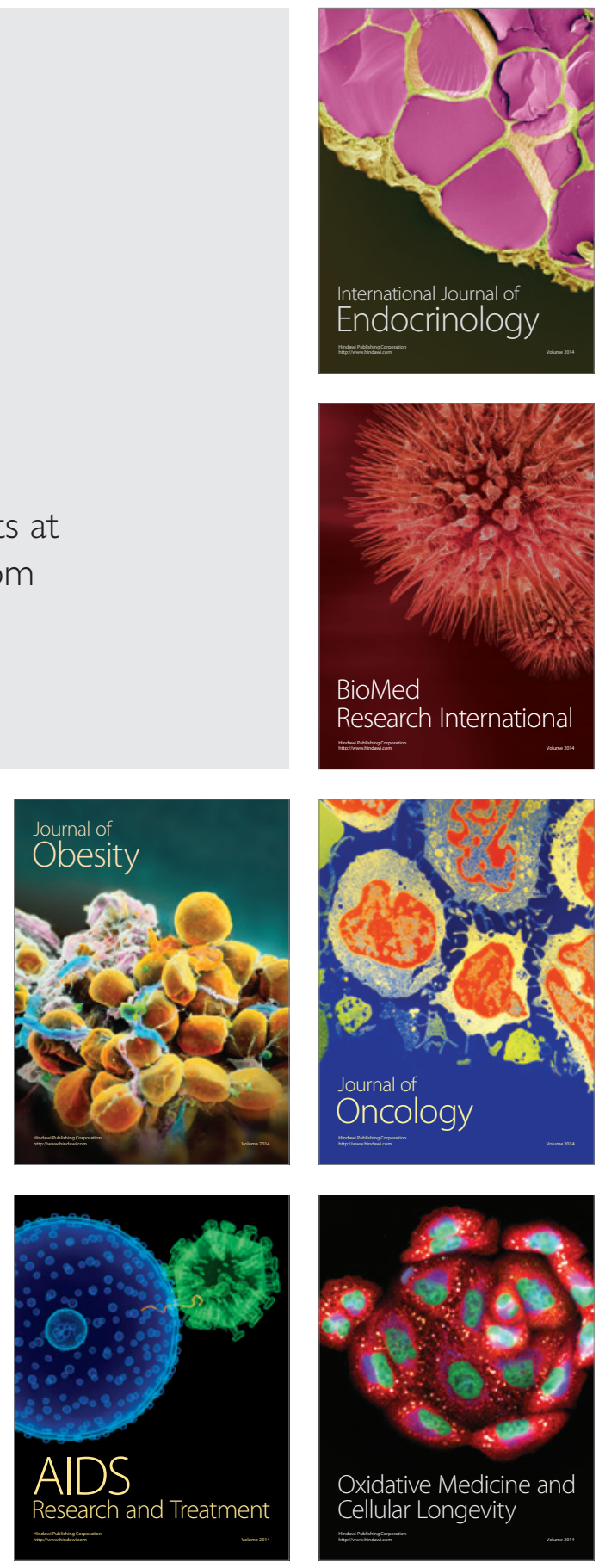